\title{
Structural changes of cerebellum and brainstem in migraine without aura
}

\author{
Zhaoxia Qin ${ }^{1 \dagger}$, Xin-Wei He ${ }^{2,3 \dagger}$, Jilei Zhang ${ }^{1}$, Shuai Xu', Ge-Fei Li ${ }^{2,3}$, Jingjing Su², Yan-Hui Shi ${ }^{2,3}$, Shiyu Ban ${ }^{1}$,

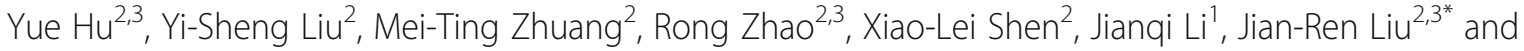 \\ Xiaoxia $\mathrm{Du}^{1 *}$ (D)
}

\begin{abstract}
Background: Increasing evidence has suggested that the cerebellum is associated with pain and migraine. In addition, the descending pain system of the brainstem is the major site of trigeminal pain processing and modulation and has been discussed as a main player in the pathophysiology of migraine. Cerebellar and brainstem structural changes associated with migraineurs remain to be further investigated.

Methods: Voxel-based morphometry (VBM) (50 controls, 50 migraineurs without aura (MWoAs)) and diffusion tensor imaging (DTI) (46 controls, 46 MWoAs) were used to assess cerebellum and brainstem anatomical alterations associated with MWoAs. We utilized a spatially unbiased infratentorial template toolbox (SUIT) to perform cerebellum and brainstem optimized VBM and DTI analysis. We extracted the average diffusion values from a probabilistic cerebellar white matter atlas to investigate whether MWoAs exhibited microstructure alterations in the cerebellar peduncle tracts.

Results: MWoAs showed decreased fractional anisotropy (FA) in the vermis $\mathrm{VI}$ extending to the bilateral lobules $\mathrm{V}$ and $\mathrm{VI}$ of the cerebellum. We also found higher axial diffusivity (AD), mean diffusivity (MD), and radial diffusivity (RD) in the right inferior cerebellum peduncle tract in MWoAs. MWoAs exhibited both reduced gray matter volume and increased $A D, M D$ and $R D$ in the spinal trigeminal nucleus (SpV).

Conclusion: MWoAs exhibited microstructural changes in the cerebellum and the local brainstem. These structural differences might contribute to dysfunction of the transmission and modulation of noxious information, trigeminal nociception, and conduction and integration of multimodal information in MWoAs. These findings further suggest involvement of the cerebellum and the brainstem in the pathology of migraine without aura.
\end{abstract}

Keywords: Migraine, cerebellum, brainstem, diffusion tensor images, voxel-based morphometry

\section{Introduction}

Migraine is a common and disabling neurological disorder that manifests as moderate to severe intensity headaches generally combined with nausea, vomiting, and hypersensitivity to visual, auditory, and olfactory stimuli [1,2]. Migraine, which affects nearly 1 billion

\footnotetext{
* Correspondence: liujr021@sjtu.edu.cn; xxdu@phy.ecnu.edu.cn

${ }^{\dagger}$ Zhaoxia Qin and Xin-Wei He contributed equally to this work.

${ }^{2}$ Department of Neurology and Jiuyuan Municipal Stroke Center, Shanghai Ninth People's Hospital, Shanghai Jiao Tong University School of Medicine, 639 Zhizaoju Road, 200011 Shanghai, People's Republic of China

'Shanghai Key Laboratory of Magnetic Resonance and Department of

Physics, School of Physics and Electronic Science, East China Normal

University, 3663 North Zhong-Shan Road, 200062 Shanghai, People's

Republic of China

Full list of author information is available at the end of the article
}

people worldwide, especially women, has become a major public health concern $[3,4]$. Therefore, approaches to better understand the pathophysiology of migraine may aid in developing better diagnostic and treatment plans for these patients.

In the past decade, increasing evidence has suggested that the cerebellum is associated with pain and migraine [5-9]. The cerebellum is a highly-organized brain area located in the hindbrain dorsal to the brainstem, which has been proposed to be related to the processing of sensorimotor, affective and cognitive information [1012]. Recent studies also demonstrated the involvement of the cerebellum in human nociception [13], and even suggest a modulating role in pain perception [14]. 
Several previous studies have reported that migraineurs exhibit functional abnormalities in the cerebellum $[8,15$, 16]. Migraine research also found an increased prevalence of ischemic lesions particularly in the cerebellar posterior lobe of migraineurs [17]. Furthermore, the cerebellum exhibited remarkably high concentrations of calcitonin gene-related peptide [18], a strong vasodilator which is a target peptide for migraine treatment [19]. Previous study found the volumes of the cerebellum and brainstem were smaller in chronic migraine compared to healthy controls[20]. Thus, there is ample evidence that the cerebellum plays significant role in the pathology of migraine. However, cerebellar anatomy alterations associated with migraineurs need further investigated. Using brainstem and cerebellar specific analysis techniques to make more accurate segmentation and spatial normalization is essential [21].

The cerebellar peduncles are major white matter tracts that communicate information among the cerebellum, the cerebral cortex, and the spinal cord. A recent study found that migraine patients have less superior cerebellar peduncle volume and that migraineurs with lower heat pain thresholds have smaller superior cerebellar peduncles [22]. These studies suggest that altered structure volume of the cerebellar peduncles is associated with migraine, while the diffusion characteristics of cerebellar peduncle tracts require further investigation. The white matter lesions of the cerebellar peduncle tracts may disconnect these relay stations in the cerebrocerebellar circuitry, eventually leading to this complex disorder. Thus, the diffusion characteristics of the cerebellar peduncles in migraineurs should be further investigated.

The descending pain system of the brainstem is the major site of trigeminal pain processing and modulation and has been discussed as a main player in the pathophysiology of migraine. Human brain imaging studies have shown that, during a migraine attack, activity increases in brainstem nuclei such as spinal trigeminal nucleus $(\mathrm{SpV})$, the dorsal pons and the midbrain periaqueductal gray matter [23, 24]. A number of studies have also reported altered sensitivity to somatosensory stimuli in the brainstem of migraine patients[2]. Functional connectivity analysis showed enhanced functional coupling between the hypothalamus and the $\mathrm{SpV}$ during the preictal phase compared with during the interictal phase [25]. Several brain morphology studies have shown that migraine is associated with decreased gray matter volume (GMV) and diffusivity abnormalities in areas involved in pain processing in the brainstem [26, 27]. Brainstem structural alterations, which might underlie the functional pathology of migraines, should be further investigated using advanced techniques.

Diedrichsen presents the Spatially Unbiased Infratentorial (SUIT) template of human brainstem and cerebellum, it allows for an improved voxel-by-voxel normalization for functional MRI analysis [21]. In this study, we used voxel-based morphometry of T1weighted anatomical and diffusion weighted images, in combination with the brainstem and cerebellum template from Diedrichsen [21] to test the following hypotheses 1) migraineurs without aura (MWoAs) may exhibited altered GMV at the cerebellum, brainstem; 2) MWoAs may displays altered diffusion characters at cerebellum, cerebellar peduncles tracts and brainstem; 3) these structural alterations may associated with disease severity, such as pain intensity, disease duration, attack frequency, Migraine Disability Assessment Scale (MIDAS) and Headache Impact Test (HIT-6) scores.

\section{Materials and methods}

\section{Subjects}

Patients were recruited from the outpatient clinic of the Department of Neurology at Shanghai Ninth People's Hospital and diagnosed with migraine without aura by a neurologist based on the International Classification of Headache Disorders 3rd edition criteria [1]. We compared voxel-based morphometric of T1-weighted anatomical images between $50 \mathrm{MWoAs}$ (mean $\pm \mathrm{SD}$ age $=$ $38.7 \pm 11.2$ years) and 50 age- and gender-matched healthy controls (HCs) (mean $\pm \mathrm{SD}$ age $=39.5 \pm 11.3$ years). Because four MWoAs did not complete the diffusion tensor imaging (DTI) scans, we compared the diffusion tensor images of $46 \mathrm{MWoAs}(38.8 \pm 11.6)$ and 46 HCs (39.0 \pm 11.2$)$. We obtained demographic and clinical data, including age, sex, disease duration, attack frequency (times/month), attack duration (hours) and pain intensity of migraine attacks assessed by a visual analogue scale (VAS)[28], of the MWoAs from the neurologist. Patients also completed the MIDAS[29] and HIT-6[30] to obtain an accurate assessment of their headache-related disability. In this study, all MWoAs were scanned during an interictal period, that is, at least no migraine attacks had occurred 48 hours before or 24 hours after MRI scanning. Migraineurs reported that they did not take preventive medication and did not have chronic migraine. The HCs had no headaches or chronic pain disorders in the past year and no neurological, psychiatric diseases, metabolic disease (e. g. diabetes mellitus) or cardiovascular disease. Moreover, the immediate family members of the HCs did not suffer from migraine or other headaches. Participants were all right handed and did not report drug abuse. The demographic and clinical data are provided in Table 1.

\section{MRI acquisition}

Magnetic resonance imaging (MRI) was performed on a 3.0 Tesla Siemens Trio Tim system equipped with a 12- 
Table 1 Demographic data and clinical scores of the migraine and control groups.

\begin{tabular}{llll}
\hline & $\begin{array}{l}\text { Migraine group } \\
(\text { Mean } \pm \text { SD) }\end{array}$ & $\begin{array}{l}\text { Control group } \\
\text { (Mean } \pm \text { SD) }\end{array}$ & $P$ value \\
\hline $\mathrm{N}$ & 50 & 50 & 1 \\
Sex (male) & $30 \%$ & $30 \%$ & 1 \\
Age (years) & $38.7 \pm 11.2$ & $39.5 \pm 11.3$ & 0.71 \\
$\begin{array}{l}\text { Disease duration } \\
\text { (years) }\end{array}$ & $8.6 \pm 6.2$ & - & - \\
$\begin{array}{l}\text { Attack duration } \\
\text { (hours) }\end{array}$ & $17.3 \pm 19.5$ & - & - \\
$\begin{array}{l}\text { Attack frequency } \\
\text { (times/months) }\end{array}$ & $3.3 \pm 2.8$ & - & - \\
Pain intensity VAS & $7.1 \pm 1.9$ & - & - \\
MIDAS & $19.6 \pm 25.7$ & - & - \\
HIT-6 & $60.5 \pm 11.7$ & - &
\end{tabular}

VAS Visual analogue scale, MIDAS Migraine disability assessment scale, HIT-6 Headache impact test, - No data

channel head coil at the Shanghai Key Laboratory of Magnetic Resonance (East China Normal University, Shanghai, China). The subjects were instructed not to move during the scans, and a Siemens dedicated filler was used to prevent head movement. High-resolution T1-weighted anatomical images were acquired by using a 3-dimensional magnetization-prepared rapid-acquisition gradient-echo pulse sequence (repetition time, 2530 $\mathrm{ms}$; echo time, $2.34 \mathrm{~ms}$; inversion time, $1100 \mathrm{~ms}$; flip angle, $7^{\circ}$; number of slices, 192; sagittal orientation; field of view, $256 \times 256 \mathrm{~mm}^{2}$; and voxel size, $1 \times 1 \times 1 \mathrm{~mm}^{3}$ ). The DTI acquisition utilized a single-shot spin-echo planar imaging sequence in the contiguous axial plane with the following parameters: repetition time, $8900 \mathrm{~ms}$; echo time, $86 \mathrm{~ms}$; b-values, 0 and $1000 \mathrm{~s} / \mathrm{mm}^{2}$; diffusion direction, 64; number of slices, 70; field of view, $256 \times 256$ $\mathrm{mm}^{2}$; and voxel size, $2 \times 2 \times 2 \mathrm{~mm}^{3}$.

\section{VBM analysis}

The cerebellum and brainstem optimized voxel-based analysis was performed using the spatially unbiased infratentorial template (SUIT) [21] toolbox implemented in Statistical Parametric Mapping software, version 12 (spm12, http://www.fil.ion.ucl.ac.uk/spm). We cropped and masked each T1 image before reslicing the image into the SUIT space so that no supratentorial gray matter (GM) could bias the results. After visually checking for data artifacts and setting the image origin at the anterior commissure of each subject, we isolated the cerebellum and brainstem structures from the surrounding tissues. To exclude any tissue outside the brainstem or the cerebellum, we hand-corrected the isolated maps using MRIcron software (http://people.cas.sc.edu/rorden/mricron). The subsequent spatial normalization and reslicing yielded cerebellar and brainstem "maps" of GM probabilities modulated by volume changes due to normalization using the 'preserve' option. The maps were resliced into the Montreal Neurological Institute (MNI) space, and a small smoothing kernel $(3 \mathrm{~mm}$ full width-at-half-maximum) was used to maintain spatial accuracy in small brainstem sites. Then, we conducted a voxelwise two-sample $\mathrm{T}$ test with sex and age as covariates to compare GMV between the two groups. The threshold was $\mathrm{p}<0.001$, two-tailed at the voxel level, and family wise error (FWE)-corrected to $\mathrm{p}<0.05$ at the cluster level. We used Duvernoy's Atlas of the Human Brainstem and Cerebellum [31] to identify the locations of significantly different clusters overlaid onto the SUIT brainstem template.

\section{DTI analysis}

DTI data were preprocessed and analyzed using the FMRIB's Diffusion Toolbox in FSL software (FMRIB Software Library, http://www.fmrib.ox.ac.uk/fsl). The original data were corrected for the effects of head movement and eddy currents using the eddy correct command by applying affine alignment of each diffusion-weighted image to the first $b=0$ image. The diffusion tensor was calculated from the images using a linear model, and then, fractional anisotropy (FA), axial diffusivity (AD), mean diffusivity (MD) and radial diffusivity (RD) whole-brain maps were derived. These images were then coregistered to each individual subject's T1-weighted image, and then, the cerebellum and brainstem were isolated, spatially normalized and resliced to the SUIT template using the parameters derived from the aforementioned T1-weighted SUIT analysis. This process resulted in cerebellum and brainstem maps of diffusion values, spatially normalized in the MNI space with raw intensities preserved (nonmodulated). The images were smoothed (3 mm full-width-at-half-maximum), and we used SPM software to conduct a voxelwise two-sample $\mathrm{T}$ test with sex and age as covariates to compare the diffusion characteristics between the two groups. The thresholds were $\mathrm{p}<0.001$, two-tailed at the voxel level, and FWE-corrected to $\mathrm{p}<0.05$ at the cluster level. We used Duvernoy's Atlas of the Human Brainstem and Cerebellum [31] to identify the locations of significantly different clusters overlaid onto the SUIT brainstem template.

\section{Cerebellum tract analysis}

We extracted the average diffusion values from a probabilistic cerebellar white matter atlas[32] at a $90 \%$ threshold, which included the superior, middle and inferior cerebellar peduncles (Additional file 1: Figure S1). We used a two-sample $\mathrm{T}$ test with sex and age as covariates to determine if there was a significant difference in 
diffusion characteristics between the MWoAs and the $\mathrm{HCs}$ in these tracts. Significant differences were determined according to $\mathrm{p}<0.0083$ (Bonferroni corrected for multiple comparisons, $\mathrm{p}<0.05)$.

\section{Correlation analysis}

Individual altered mean GMVs and diffusion characteristics for the surviving clusters and cerebellar tracts of the MWoAs were extracted for Spearson's correlation with the clinical data, including disease duration, attack frequency, attack duration, and VAS, MIDAS, and HIT-6 scores. Significant correlations were determined according to $\mathrm{p}<0.0083$ (Bonferroni corrected for multiple comparisons, $\mathrm{p}<0.05)$.

\section{Results}

\section{Demographic and clinical characteristics}

The demographic and clinical data of the MWoA and control groups are presented in Table 1 . There was no significant difference in age (two-sample $\mathrm{T}$ test; $\mathrm{p}>0.05$ ).

\section{Gray matter volume changes (VBM)}

A comparison of GMVs revealed a GMV decrease in the $\mathrm{SpV}$ in migraineurs relative to controls, as shown in Figure $3(\mathrm{p}<0.001$ at the voxel level, FWE-corrected $\mathrm{p}=$ 0.067 at the cluster level). The peak MNI coordinates were $(4,-45,-65)$.

\section{Changes in diffusion characteristics (DTI)}

Compared with controls, MWoAs had decreased FA at the vermis VI extending to the bilateral lobule V and the bilateral lobule VI of the cerebellum (Table 2, Fig. 1). Cerebellar tract analysis revealed that MWoAs had higher $\mathrm{AD}, \mathrm{MD}$ and $\mathrm{RD}$ in the right inferior cerebellum peduncle (ICP) (Table 3, Fig. 2). MWoAs had higher AD, MD, and $\mathrm{RD}$ in the region of the SpV (Table 2, Fig. 3).

\section{Correlation with clinical scores}

Altered GMVs and diffusion characteristics were not significantly correlated with MWoA clinical data (Table 4).

\section{Discussion}

In the present study, we used the SUIT toolbox to explore alterations in GMVs and diffusion properties of the cerebellum and the brainstem associated with MWoAs. We found decreased FA properties in the vermis VI extending to the bilateral lobule V and the bilateral lobule VI of the cerebellum. Higher AD, MD, and $\mathrm{RD}$ were found in the right inferior cerebellum peduncle tract in MWoAs. We also found both reduced GMV and increased $\mathrm{AD}, \mathrm{RD}$, and $\mathrm{MD}$ with regard to microstructural alterations that occurred in all divisions of the SpV. In migraine subjects, these GMV and diffusivity changes were not correlated with migraine frequency, duration or other clinical data, which may due to the limited number of patients. In the other side, we think that repeated, long-term headache attacks may induce adaptive or maladaptive changes, and such changes would be highly complex and nonlinear.

We found that MWoAs exhibited decreased FA in the vermis VI extending to the bilateral lobule V and the bilateral lobule VI compared with HCs. FA is a parameter that can be used to investigate anisotropy and is a marker of the change in shape of the diffusion ellipsoid, which is highly sensitive to microstructural alterations[33]. Our results showed that MWoA-associated cerebellar microstructure alterations occurred at the vermis VI and at lobules V and VI. Stimulation of rat cerebellar cortex at posterior vermis (lobe VI), increases neural responses to a noxious visceral stimulus in and around the termination sites of nociceptive afferents in the spinal cord[34], which reflect cerebellar stimulation modulates nociception. In addition, a recent study found that trigeminal nociception is processed in the cerebellar lobules V and VI ipsilateral to the nociceptive stimulus[35]. An individual's intensity and unpleasantness

Table 2 Brain regions with significant differences in diffusion characteristics between MWoAs and healthy controls.

\begin{tabular}{|c|c|c|c|c|c|c|}
\hline & \multirow[t]{2}{*}{ Cluster size } & \multirow[t]{2}{*}{ Peak T value } & \multicolumn{3}{|c|}{ MNI coordinates } & \multirow{2}{*}{$\begin{array}{l}\text { Cluster level } \\
p_{F W E-\text { corr }}\end{array}$} \\
\hline & & & $x$ & y & z & \\
\hline \multicolumn{7}{|l|}{ FA: (Migraine group < Control group) } \\
\hline Vermis VI extending to the bilateral lobule V and bilateral lobule VI & 163 & 4.18 & 6 & -68 & -17 & 0.002 \\
\hline \multicolumn{7}{|l|}{ AD: (Migraine group > Control group) } \\
\hline SpV & 123 & 4.72 & -2 & -42 & -63 & 0.026 \\
\hline \multicolumn{7}{|l|}{ MD: (Migraine group > Control group) } \\
\hline SpV & 170 & 5.00 & -4 & -42 & -65 & 0.006 \\
\hline \multicolumn{7}{|l|}{ RD: (Migraine group > Control group) } \\
\hline SpV & 173 & 5.08 & -4 & -42 & -65 & 0.004 \\
\hline
\end{tabular}

The results were assigned thresholds at $\mathrm{p}<0.001$ (voxel level) and FWE-corrected to $\mathrm{p}<0.05$ at the cluster level. $A D$ Axial diffusivity, $M D$ Mean diffusivity, $R D$ Radial diffusivity, FA Fractional anisotropy, SpV Spinal trigeminal nucleus 


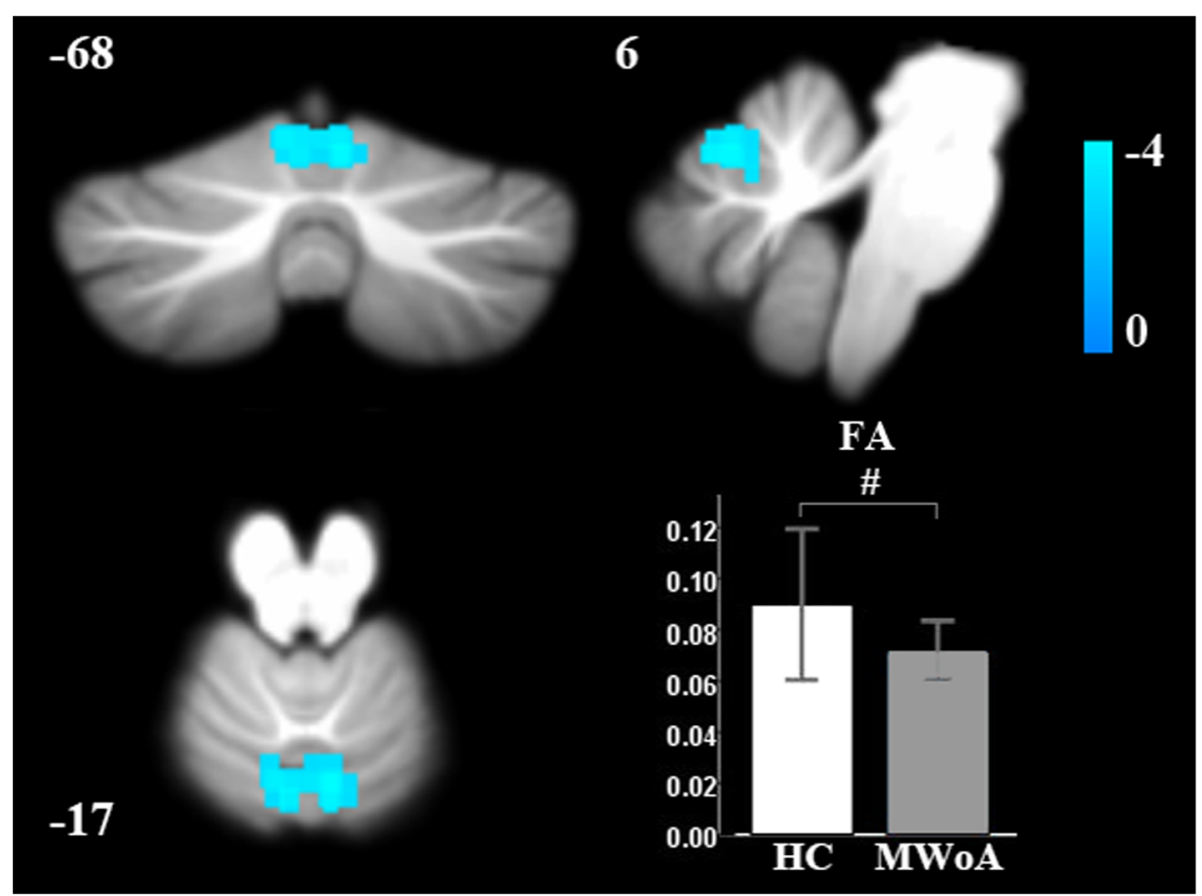

Fig. 1 Brain regions with significantly decreased fractional anisotropy (FA) of MWoAs relative to healthy controls. " Statistical significance of $p<0.001$ at the voxel level, FWE-corrected $p<0.05$ at the cluster level.

ratings are processed in the hemispheric lobule VI and extending to lobule V[35]. Importantly, lobule VI has been demonstrated to be involved in the integration of information across multiple modalities[36]. Patients' microstructure alterations at the vermis VI and at lobules V and VI might have an impact on the trigeminal nociception and multimodal information integration, which could contribute to the pathology of MWoA.

We also found that MWoAs exhibited significantly higher $\mathrm{AD}, \mathrm{MD}$, and $\mathrm{RD}$ at the right $\mathrm{ICP}$ relative to

Table 3 Diffusion characteristics for 3 major cerebellar tracts in the MWoA and healthy controls.

\begin{tabular}{|c|c|c|c|c|c|c|}
\hline & L-SCP & R-SCP & L-MCP & R-MCP & L-ICP & R-ICP \\
\hline \multicolumn{7}{|l|}{$A D$} \\
\hline $\mathrm{HC}$ & $0.92 \pm 0.14$ & $0.91 \pm 0.14$ & $0.83 \pm 0.12$ & $0.86 \pm 0.12$ & $0.90 \pm 0.14$ & $0.89 \pm 0.14$ \\
\hline MWoA & $0.94 \pm 0.10$ & $0.96 \pm 0.10$ & $0.81 \pm 0.08$ & $0.84 \pm 0.08$ & $0.96 \pm 0.10$ & $0.97 \pm 0.11$ \\
\hline$P$ value & 0.590 & 0.069 & 0.324 & 0.462 & 0.045 & $0.002^{*}$ \\
\hline \multicolumn{7}{|l|}{ MD } \\
\hline $\mathrm{HC}$ & $0.63 \pm 0.11$ & $0.62 \pm 0.10$ & $0.57 \pm 0.14$ & $0.59 \pm 0.13$ & $0.70 \pm 0.12$ & $0.69 \pm 0.11$ \\
\hline MWoA & $0.63 \pm 0.07$ & $0.66 \pm 0.08$ & $0.52 \pm 0.05$ & $0.55 \pm 0.05$ & $0.75 \pm 0.08$ & $0.76 \pm 0.09$ \\
\hline$P$ value & 0.997 & 0.072 & 0.032 & 0.044 & 0.057 & $0.001^{*}$ \\
\hline \multicolumn{7}{|l|}{$\mathrm{RD}$} \\
\hline $\mathrm{HC}$ & $0.48 \pm 0.10$ & $0.48 \pm 0.09$ & $0.44 \pm 0.15$ & $0.45 \pm 0.15$ & $0.60 \pm 0.11$ & $0.59 \pm 0.10$ \\
\hline MWoA & $0.47 \pm 0.06$ & $0.51 \pm 0.07$ & $0.38 \pm 0.04$ & $0.40 \pm 0.04$ & $0.64 \pm 0.07$ & $0.65 \pm 0.08$ \\
\hline$P$ value & 0.694 & 0.094 & 0.013 & 0.015 & 0.079 & $0.001^{*}$ \\
\hline \multicolumn{7}{|l|}{ FA } \\
\hline $\mathrm{HC}$ & $0.30 \pm 0.06$ & $0.30 \pm 0.05$ & $0.37 \pm 0.12$ & $0.37 \pm 0.12$ & $0.22 \pm 0.05$ & $0.21 \pm 0.05$ \\
\hline MWoA & $0.31 \pm 0.04$ & $0.31 \pm 0.04$ & $0.41 \pm 0.05$ & $0.41 \pm 0.05$ & $0.23 \pm 0.03$ & $0.23 \pm 0.03$ \\
\hline$P$ value & 0.157 & 0.162 & 0.012 & 0.011 & 0.094 & 0.125 \\
\hline
\end{tabular}

$A D$ Axial diffusivity, MD Mean diffusivity, $R D$ Radial diffusivity, FA Fractional anisotropy, SCP Superior cerebellar peduncle, $M C P$ Middle cerebellar peduncle, ICP Inferior cerebellar peduncle, * Statistical significance was set at $p<0.0083$, (Bonferroni corrected $p<0.05$ ). The MD, RD, and AD metrics are shown as $10^{-3}$, and FA is shown as the actual value. Note that only the right inferior cerebellar peduncle displayed significant increases in MD, RD, and AD compared with controls. 


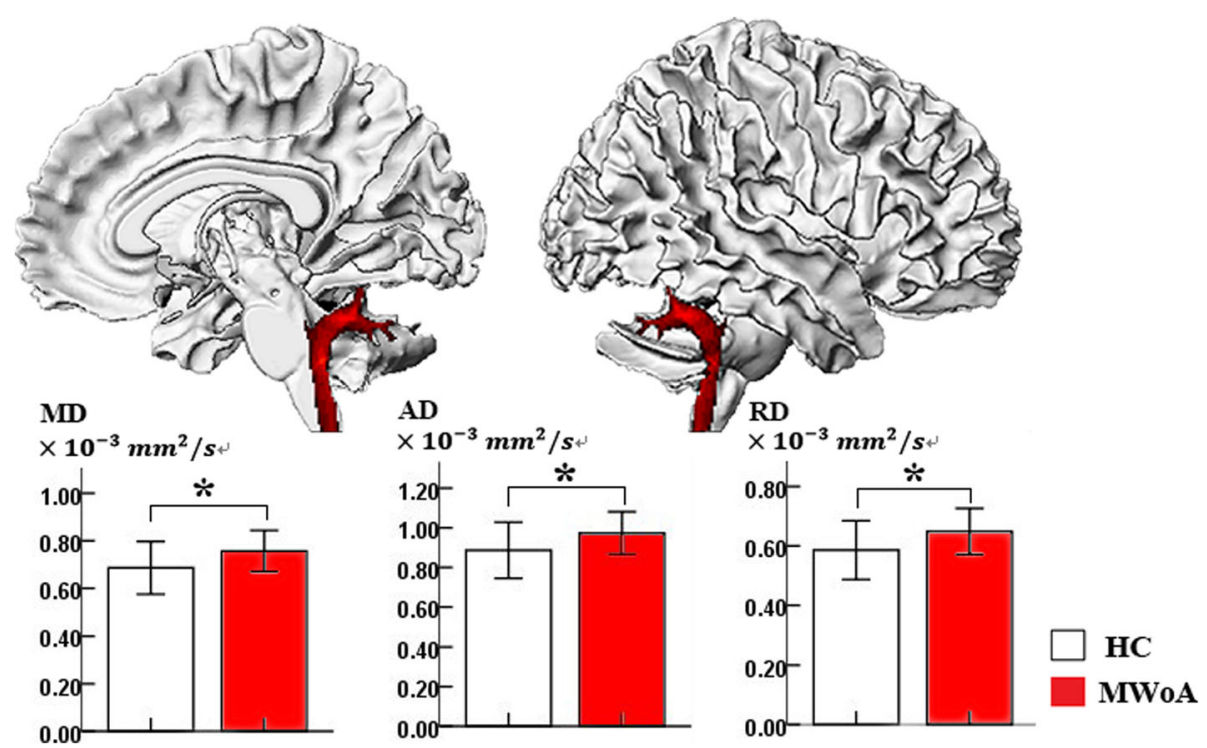

Fig. 2 Sagittal view of the right inferior cerebellar peduncle with significantly increased mean diffusivity (MD), axial diffusivity (AD), and radial diffusivity (RD) in MWoAs relative to healthy controls; *Statistical significance was $p<0.0083$ (Bonferroni corrected for multiple comparisons $\mathrm{p}<0.05)$.

HCs, which suggested that MWoAs exhibited altered microstructure of the ICP tract. The ICPs contain both afferent and efferent pathways and connect the medulla to the cerebellum[37]. The ICP carries many types of input and output fibers that are mainly related to integrating proprioceptive sensory input with motor vestibular functions, such as balance and posture maintenance[37]. The ICP connects the inferior olive to the cerebellum, and the inferior olive receives descending inputs from the parvicellular red nucleus, which in turn receives inputs from the primary motor cortex, the supplementary motor cortex, the premotor cortex, the primary somatosensory cortex, and the superior parietal lobe[38]. Based on these connections, the red nucleus-inferior olive system seems to convey motor and some sensory efferents to the cerebellum[38]. In addition, cerebellar anterior lobules receive sensorimotor input from the body through the ICPs [39]. Thus, the altered microstructure

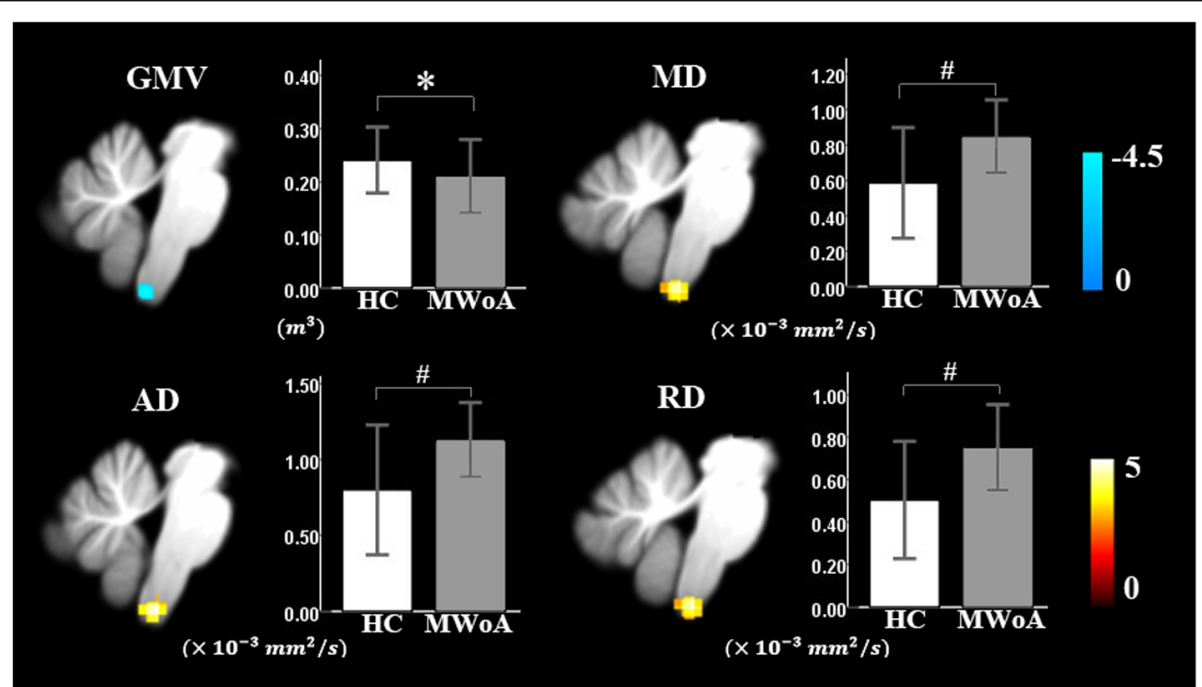

Fig. 3 Sagittal view of the spinal trigeminal nucleus (SpV) with significant differences in gray matter volume and diffusion characteristics between MWoAs and healthy controls. * Statistical significance of $p<0.001$ at the voxel level, FWE-corrected $p<0.067$ at the cluster level; \# Statistical significance of $p<0.001$ at the voxel level, FWE-corrected $p<0.05$ at the cluster level. 
Table 4 Clinical correlations with altered GMVs and diffusion characteristics of patients with MWoA.

\begin{tabular}{|c|c|c|c|c|c|c|}
\hline & Disease duration (years) & Duration (hours) & Frequency (times/ months) & Pain intensity VAS & MIDAS & HIT-6 \\
\hline \multicolumn{7}{|c|}{ Decreased GMVs at SpV } \\
\hline r & 0.119 & 0.169 & -0.249 & 0.044 & -0.221 & 0.038 \\
\hline$P$ value & 0.432 & 0.261 & 0.096 & 0.773 & 0.140 & 0.800 \\
\hline \multicolumn{7}{|c|}{ Decreased FA at Vermis VI extending to the bilateral lobule V and bilateral lobule VI } \\
\hline r & -0.047 & -0.250 & 0.347 & 0.006 & 0.190 & 0.110 \\
\hline$P$ value & 0.759 & 0.094 & 0.018 & 0.970 & 0.206 & 0.465 \\
\hline \multicolumn{7}{|c|}{ Increased AD at R-ICP } \\
\hline r & -0.058 & -0.125 & 0.160 & 0.218 & 0.106 & 0.120 \\
\hline$P$ value & 0.703 & 0.407 & 0.289 & 0.146 & 0.485 & 0.425 \\
\hline \multicolumn{7}{|c|}{ increased MD at R-ICP } \\
\hline r & -0.105 & -0.072 & 0.139 & 0.147 & 0.064 & 0.050 \\
\hline$P$ value & 0.486 & 0.633 & 0.355 & 0.330 & 0.671 & 0.741 \\
\hline \multicolumn{7}{|c|}{ increased RD at R-ICP } \\
\hline r & -0.155 & -0.051 & 0.123 & 0.114 & 0.065 & 0.033 \\
\hline$P$ value & 0.305 & 0.734 & 0.416 & 0.449 & 0.670 & 0.827 \\
\hline \multicolumn{7}{|c|}{ increased $A D$ at SpV } \\
\hline r & -0.246 & 0.034 & 0.107 & -0.023 & -0.050 & -0.135 \\
\hline$P$ value & 0.099 & 0.823 & 0.479 & 0.881 & 0.740 & 0.370 \\
\hline \multicolumn{7}{|c|}{ increased MD at SpV } \\
\hline r & -0.234 & 0.053 & 0.105 & -0.036 & -0.038 & -0.165 \\
\hline$P$ value & 0.118 & 0.728 & 0.486 & 0.814 & 0.803 & 0.273 \\
\hline \multicolumn{7}{|c|}{ increased RD at SpV } \\
\hline r & -0.250 & 0.087 & 0.106 & -0.044 & -0.041 & -0.162 \\
\hline$P$ value & 0.094 & 0.566 & 0.482 & 0.770 & 0.786 & 0.283 \\
\hline
\end{tabular}

VAS Visual analogue scale, MIDAS Migraine disability assessment scale, HIT-6 Headache impact test; Frequency: the average number of attacks per month in the last three month; $A D$ Axial diffusivity, MD Mean diffusivity, RD Radial diffusivity, FA Fractional anisotropy, SCP Superior cerebellar peduncle, $M C P$ Middle cerebellar peduncle, ICP Inferior cerebellar peduncle, SpV Spinal trigeminal nucleus, $r$ Spearson's rho; Significant correlations were determined according to $p<0.0083$ (Bonferroni corrected for multiple comparisons, $\mathrm{p}<0.05)$.

of the ICP tract suggests that MWoAs may exhibit dysfunctional conduction and integration of sensorimotor information at the medulla to cerebellum level, which could lead to higher-order dysfunction of cerebral cortex pain modulation and may be associated with the pathological mechanism of migraine without aura. In the future work, the microstructure of fiber tract connecting cerebellum to cerebral cortex remain to be further explored.

Previous study found the volumes of the cerebellum and brainstem were smaller in chronic migraineurs than healthy controls [20]. In our study, consistent with Marciszewski's study [27], reduced GMV and increased MD were found in the $\mathrm{SpV}$ in MWoAs. In addition, we found increased $\mathrm{AD}$ and $\mathrm{RD}$ in the $\mathrm{SpV}$, which suggested more subtle changes in tissue microstructure. These changes may result from loss of fiber tracts and disruption of the myelin sheath, increased membrane permeability, destruction of intracellular compartments, and glial alterations[40,41]. The reduced GMV of the
$\mathrm{SpV}$ in MWoAs may result from shrinkage or atrophy of neurons or glia or synaptic loss[42]. The $\mathrm{SpV}$ is a key structure in the brainstem involved in migraine pathophysiology, and it receives information from the trigeminal ganglion cells innervating the meninges and the cranial vasculature. Furthermore, the $\mathrm{SpV}$ is known to innervate several migraine-relevant structures, such as the thalamus and the hypothalamus [43]. Numerous human studies have suggested that the $\mathrm{SpV}$ plays an important role in the pathology of migraine. According to Stankewitz et al., the activity of the SpV in response to nociceptive stimulation predicted the time to the next headache [44]. Migraine subjects also showed greater amplification from the $\mathrm{SpV}$ to the posterior insula and the hypothalamus than controls [45]. Our results further supported that MWoAs have reduced GMV at the SpV and diffusion alterations of the $\mathrm{SpV}$ in three dimensions. These findings suggest that structural changes in the $\mathrm{SpV}$ may contribute to dysfunction of the SpV in transmitting noxious information from the cranial vessels and 
the meninges as well as atypical modulation of noxious inputs. Anatomical rat and human studies have described direct descending projections from the cerebral cortex to the $\mathrm{SpV}$ [46] and indirect projections arising in the cerebral cortex that project via the hypothalamus to the SpV [47]. Structural changes in the SpV might leading to higher-order dysfunction of cerebral cortex pain modulation.

Although our research revealed that MWoAs exhibit anatomical changes at the brainstem, the cerebellum and the cerebellar peduncles, the current study had several limitations. First, we examined patients only in the interictal phase, and therefore, brain structure in the ictal phase must also be explored. Second, we focused on anatomical changes in MWoAs but did not examine migraineurs with aura and chronic migraineurs. In a future study, we will evaluate the structural abnormalities of the brainstem and cerebellar areas of migraineurs with aura and chronic migraineurs during the ictal phase.

\section{Conclusion}

In the present study, we found decreased FA in the vermis VI extending to the bilateral lobules V and VI of the cerebellum, which may contribute to the dysfunction of trigeminal nociception and multimodal information integration in MWoAs. We also found higher $\mathrm{AD}, \mathrm{MD}$, and $\mathrm{RD}$ in the right inferior cerebellum peduncle tract, which suggests that MWoAs exhibit atypical conduction and integration of sensorimotor information at the medulla to cerebellum level. MWoAs exhibited both reduced GMV and increased $\mathrm{AD}, \mathrm{RD}$, and $\mathrm{MD}$ in the $\mathrm{SpV}$, which further strengthens the evidence linking migraine without aura to the SpV. Structural changes in the SpV could contribute to the dysfunction of the $\mathrm{SpV}$ in transmitting and modulating noxious information from the cranial vessels and the meninges.

\section{Additional file}

Additional file 1: Figure S1. The probabilistic cerebellar white matter atlas (Van Baarsen et al., 2016, including the superior, middle and inferior cerebellar peduncles, at a threshold of 90\%). (TIF $1124 \mathrm{~kb}$ )

\section{Abbreviations}

AD: Axial diffusivity; DTI: Diffusion tensor imaging; FA: Fractional anisotropy; FWE: Familywise error; GM: Gray matter; GMV: Gray matter volume; HCs: Healthy controls; HIT-6: Headache Impact Test; ICP: Inferior cerebellum peduncle; MD: Mean diffusivity; MIDAS: Migraine Disability Assessment Scale; MNI: Montreal Neurological Institute; MRl: magnetic resonance imaging; MWoAs: Migraineurs without aura; RD: Radial diffusivity; SpV: Spinal trigeminal nucleus; SUIT: Spatially Unbiased Infra-tentorial; VAS: Visual analogue scale; VBM: Voxel based morphometry

\section{Acknowledgments}

We thank the patients who participated in the study for their support.

\section{Authors' contributions}

$X D, J L$ and ZQ designed the study. XH, ZQ, JZ, SX, GL, JS. YS, SB, YH, YL, MZ, $R Z$ and $X S$ performed the experiments. $Z Q, J Z, J L$ and $S X$ analysed the data. $X D, J L$ and $Z Q$ prepared the manuscript. All authors read and approved the final manuscript.

\section{Funding}

This research was supported by grants from the National Natural Science Foundation of China (Nos. 81571658 to X. X. Du), the National Natural Science Foundation of China (81271302 to J.R. Liu), a research innovation project of the Shanghai Municipal Science and Technology Commission (No. 14JC1404300, to J.R. Liu), the "Prevention and Control of Chronic Diseases Project" of Shanghai Hospital Development Center (No. SHDC12015310, to J.R. Liu), a project of the SHSMU-ION Research Center for Brain Disorders (No. 2015NKX006, to J.R. Liu), a project of the Shanghai Municipal Education Commission-Gaofeng Clinical Medicine Grant Support (No. 20161422 to J. R. Liu), a Clinical Research Project of Shanghai Jiao Tong University School of Medicine (No. DLY201614 to J.R. Liu), and the Biomedicine Key program of the Shanghai Municipal Science and Technology Commission (No. 16411953100 to J.R. Liu).

\section{Availability of data and materials}

The datasets generated during and/or analysed during the current study are available from the corresponding author on reasonable request.

\section{Ethics approval and consent to participate}

This study was conducted according to the Declaration of Helsinki, and written informed consent was obtained from all participants. This study was approved by the East China Normal University Committee on Human Research (Project No. HR2015/03011) and by the Independent Ethics Committee of Shanghai Ninth People's Hospital (Project No. [2016]01).

\section{Consent for publication}

Not applicable.

\section{Competing interests}

The authors declare no potential conflicts of interest with respect to the research, authorship, and/or publication of this article.

\section{Author details}

${ }^{1}$ Shanghai Key Laboratory of Magnetic Resonance and Department of Physics, School of Physics and Electronic Science, East China Normal University, 3663 North Zhong-Shan Road, 200062 Shanghai, People's Republic of China. ${ }^{2}$ Department of Neurology and Jiuyuan Municipal Stroke Center, Shanghai Ninth People's Hospital, Shanghai Jiao Tong University School of Medicine, 639 Zhizaoju Road, 200011 Shanghai, People's Republic of China. ${ }^{3}$ Clinical Research Center, Shanghai Jiao Tong University School of Medicine, Shanghai 200011, China.

Received: 3 July 2019 Accepted: 26 August 2019

Published online: 02 September 2019

\section{References}

1. Headache Classification Committee of the International Headache Society (IHS) The International Classification of Headache Disorders, 3rd edition. Cephalalgia : an international journal of headache 2018, 38(1):1-211.

2. Schwedt TJ, Chiang CC, Chong CD, Dodick DW (2015) Lancet Neurol 14(1): 81-91

3. Vos T, Flaxman AD, Naghavi M, Lozano R, Michaud C, Ezzati M, Shibuya K, Salomon JA, Abdalla S, Aboyans V et al (2012) Years lived with disability (YLDs) for 1160 sequelae of 289 diseases and injuries 1990-2010: a systematic analysis for the Global Burden of Disease Study 2010. Lancet 380(9859):2163-2196

4. Burch RC, Loder S, Loder E, Smitherman TA (2015) The prevalence and burden of migraine and severe headache in the United States: updated statistics from government health surveillance studies. Headache 55(1):21-34

5. Coombes SA, Misra G (2016) Pain and motor processing in the human cerebellum. Pain 157(1):117-127

6. Vincent M, Hadjikhani N (2007) The cerebellum and migraine. Headache 47(6):820-833 
7. Carpenter MB, Hanna GR (1961) Fiber projections from the spinal trigeminal nucleus in the cat. Journal of Comparative Neurology 117(1):117-131

8. Mehnert J, May A (2019) Functional and structural alterations in the migraine cerebellum. Journal of cerebral blood flow and metabolism : official journal of the International Society of Cerebral Blood Flow and Metabolism 39 (4):730-739

9. Kros L, Angueyra Aristizabal CA, Khodakhah K (2018) Cerebellar involvement in migraine. Cephalalgia : an international journal of headache 38(11):1782-1791

10. Timmann D, Drepper J, Frings M, Maschke M, Richter S, Gerwig M, Kolb FP (2010) The human cerebellum contributes to motor, emotional and cognitive associative learning. A review. Cortex 46 (7):845-857

11. Stoodley CJ, Schmahmann JD (2010) Evidence for topographic organization in the cerebellum of motor control versus cognitive and affective processing. Cortex 46(7):831-844

12. Stoodley CJ, Schmahmann JD (2009) Functional topography in the human cerebellum: a meta-analysis of neuroimaging studies. Neuroimage 44(2):489-501

13. Moulton EA, Schmahmann JD, Becerra L, Borsook D (2010) The cerebellum and pain: passive integrator or active participator? Brain research reviews 65(1):14-27

14. Ruscheweyh R, Kühnel M, Filippopulos F, Blum B, Eggert T, Straube A (2014) Altered experimental pain perception after cerebellar infarction. PAIN ${ }^{\circledR}$ 155(7):1303-1312

15. Wang M, Su J, Zhang J, Zhao Y, Yao Q, Zhang Q, Zhang H, Wang S, Li G-F, Liu J-R (2017) Visual cortex and cerebellum hyperactivation during negative emotion picture stimuli in migraine patients. Scientific reports 7:41919

16. Wang JJ, Chen X, Sah SK, Zeng C, Li YM, Li N, Liu MQ, Du SL (2016) Amplitude of low-frequency fluctuation (ALFF) and fractional ALFF in migraine patients: a resting-state functional MRI study. Clinical radiology 71(6):558-564

17. Koppen H, Boele H-J, Palm-Meinders IH, Koutstaal BJ, Horlings CG, Koekkoek BK, van der Geest J, Smit AE, van Buchem MA, Launer $\sqcup$ (2017) Cerebellar function and ischemic brain lesions in migraine patients from the general population. Cephalalgia 37(2):177-190

18. Eftekhari S, Salvatore CA, Gaspar RC, Roberts R, O'Malley S, Zeng Z, Edvinsson L (2013) Localization of CGRP receptor components, CGRP, and receptor binding sites in human and rhesus cerebellar cortex. The Cerebellum 12(6):937-949

19. Edvinsson $L$ (2015) The journey to establish CGRP as a migraine target: a retrospective view. Headache: The Journal of Head and Face Pain 55(9):1249-1255

20. Bilgiç B, Kocaman G, Arslan AB, Noyan H, Sherifov R, Alkan A, Asil T, Parman Y, Baykan B (2016) Volumetric differences suggest involvement of cerebellum and brainstem in chronic migraine. Cephalalgia 36(4):301-308

21. Diedrichsen J (2006) A spatially unbiased atlas template of the human cerebellum. Neurolmage 33 (1):127-138

22. Chong CD, Plasencia JD, Frakes DH, Schwedt TJ (2017) Structural alterations of the brainstem in migraine. Neurolmage: Clinical 13:223-227

23. Bahra A, Matharu MS, Buchel C, Frackowiak RSJ, Goadsby PJ (2001) Brainstem activation specific to migraine headache. The Lancet 357(9261): 1016-1017

24. Weiller C, May A, Va L, Jüptner M, Kaube H, Schayck R, Coenen H, Dlener H (1995) Brain stem activation in spontaneous human migraine attacks. Nature medicine 1(7):658

25. Schulte LH, Sprenger C, May A (2016) Physiological brainstem mechanisms of trigeminal nociception: An fMRI study at 3T. Neurolmage 124:518-525

26. Jia Z, Yu S (2017) Grey matter alterations in migraine: a systematic review and meta-analysis. Neurolmage: Clinical 14:130-140

27. Marciszewski KK, Meylakh N, Di Pietro F, Macefield VG, Macey PM, Henderson LA (2018) Altered brainstem anatomy in migraine. Cephalalgia 38(3):476-486

28. Hawker GA, Mian S, Kendzerska T, French M (2011) Measures of adult pain: Visual analog scale for pain (vas pain), numeric rating scale for pain (nrs pain), mcgill pain questionnaire (mpq), short-form mcgill pain questionnaire (sf-mpq), chronic pain grade scale (cpgs), short form-36 bodily pain scale (sf-36 bps), and measure of intermittent and constant osteoarthritis pain (icoap). Arthritis care \& research 63(S11):S240-S252

29. Stewart WF, Lipton RB, Whyte J, Dowson A, Kolodner K, Ja L, Sawyer J (1999) An international study to assess reliability of the Migraine Disability Assessment (MIDAS) score. Neurology 53(5):988-988
30. Kosinski M, Bayliss M, Bjorner J, Ware J, Garber W, Batenhorst A, Cady R, Dahlöf C, Dowson A, Tepper S (2003) A six-item short-form survey for measuring headache impact: The HIT-6 ${ }^{\mathrm{TM}}$. Quality of Life Research 12(8): 963-974

31. Naidich TP, Duvernoy HM, Delman BN, Sorensen AG, Kollias SS, Haacke EM (2009) Duvernoy's atlas of the human brain stem and cerebellum: high-field $\mathrm{MRI}$, surface anatomy, internal structure, vascularization and $3 \mathrm{D}$ sectional anatomy. Springer Science \& Business Media

32. Van Baarsen K, Kleinnijenhuis M, Jbabdi S, Sotiropoulos SN, Grotenhuis J, Walsum v (2016) AvC: A probabilistic atlas of the cerebellar white matter. Neurolmage 124:724-732

33. Tae W-S, Ham B-J, Pyun S-B, Kang S-H, Kim B-J (2018) Current clinical applications of diffusion-tensor imaging in neurological disorders. Journal of Clinical Neurology 14(2):129-140

34. C S, W W (2001) Nociceptive visceral stimulation modulates the activity of cerebellar Purkinje cells. Experimental brain research 140(1):122-126

35. Mehnert J, Schulte L, Timmann D, May A (2017) Activity and connectivity of the cerebellum in trigeminal nociception. Neurolmage 150:112-118

36. Christensen A, Giese MA, Sultan F, Mueller OM, Goericke SL, Ig W, Timmann $D$ (2014) An intact action-perception coupling depends on the integrity of the cerebellum. Journal of Neuroscience 34(19):6707-6716

37. Mori S, Wakana S, Van Zijl PC, Nagae-Poetscher L (2005) MRI atlas of human white matter. Elsevier

38. Schmahmann JD (1996) From movement to thought: anatomic substrates of the cerebellar contribution to cognitive processing. Human brain mapping 4(3):174-198

39. Dum RP, Strick PL (2003) An unfolded map of the cerebellar dentate nucleus and its projections to the cerebral cortex. Journal of neurophysiology 89(1):634-639

40. Song SK, Sun SW, Ramsbottom MJ, Chang C, Russell J, Cross AH (2002) Dysmyelination revealed through MRI as increased radial (but unchanged axial) diffusion of water. Neurolmage 17(3):1429-1436

41. Beaulieu C (2002) The basis of anisotropic water diffusion in the nervous system - a technical review. NMR in biomedicine 15(7-8):435-455

42. May A, Gaser C (2006) Magnetic resonance-based morphometry: a window into structural plasticity of the brain. Current opinion in neurology 19(4):407-411

43. Burstein R, Noseda R, Borsook D (2015) Migraine: multiple processes, complex pathophysiology. Journal of Neuroscience 35(17):6619-6629

44. Stankewitz A, Aderjan D, Eippert F, May A (2011) Trigeminal nociceptive transmission in migraineurs predicts migraine attacks. The Journal of neuroscience : the official journal of the Society for Neuroscience 31(6): 1937-1943

45. Lee J, Lin RL, Garcia RG, Kim J, Kim H, Loggia ML, Mawla I, Wasan AD, Edwards RR, Rosen BR (2017) Reduced insula habituation associated with amplification of trigeminal brainstem input in migraine. Cephalalgia 37(11): $1026-1038$

46. Noseda R, Constandil L, Bourgeais L, Chalus M, Villanueva L (2010) Changes of meningeal excitability mediated by corticotrigeminal networks: a link for the endogenous modulation of migraine pain. Journal of Neuroscience 30(43):14420-14429

47. Robert C, Bourgeais L, Arreto C-D, Condes-Lara M, Noseda R, Jay T, Villanueva $L$ (2013) Paraventricular hypothalamic regulation of trigeminovascular mechanisms involved in headaches. Journal of Neuroscience 33(20):8827-8840

\section{Publisher's Note}

Springer Nature remains neutral with regard to jurisdictional claims in published maps and institutional affiliations. 